\title{
Reports of Oncological Societies . Mitteilungen onkologischer Gesellschaften
}

\section{Deutsche Krebsgesellschaft e.V.}

\section{Krebs-Selbsthilfegruppen per Mausklick}

Niemand kann die Ängste und Probleme von Krebs-Patienten besser verstehen als Menschen, die selbst betroffen sind oder waren. Denn: Sie sind Experten in eigener Sache! Immer mehr Frauen und Männer mit der Diagnose Krebs finden sich deshalb in Selbsthilfegruppen zusammen. Diese Gruppen bieten den Betroffenen nicht nur die Möglichkeit, Erfahrungen auszutauschen. Hier können sich Interessierte auch über das Thema Krebs informieren - zum Beispiel über neue Therapiemöglichkeiten. Mittlerweile bestehen in der Bundesrepublik etwa 1500 Gruppen, in denen sich Krebs-Patienten und deren Angehörige engagieren. Wie findet man jedoch die geeignete Selbsthilfegruppe? Die bewährte Liste sämtlicher Selbsthilfegruppen für krebskranke Menschen in Deutschland - das Selbsthilfe-Kompendium der Deutschen Krebsgesellschaft e.V. - präsentiert sich nun in neuer Aufmachung. Um die Suche nach einer geeigneten Gruppe weiter zu erleichtern, bietet sie ihr umfassendes Kompendium ab sofort auf CD-ROM an.

Neben den Adressen der Selbsthilfegruppen enthält die CDROM zusätzlich Anschriften von Krebsberatungsstellen, Tumorzentren und Nachsorgeeinrichtungen. Damit das Adressenmaterial immer auf dem neuesten Stand ist, werden die Daten alle 6 Monate aktualisiert. Gesucht werden kann unter den Kriterien Bundesländer, Städte, Tumorlokalisation sowie unter selbstdefinierten Suchbegriffen. Die abrufbaren Informationen umfassen neben den Adressen und Telefonnummern auch Faxnummern, E-mail-Adressen und gegebenenfalls Hinweise auf Internetpräsenz. Selbstverständlich sind auch die Namen der Ansprechpartner und die Treffpunkte (Ort und Zeit) der Selbsthilfegruppen aufgeführt.

Viel Wert legte die Deutsche Krebsgesellschaft e.V. auf die Benutzerfreundlichkeit der CD-ROM: Die Daten können direkt ausgedruckt werden. Besonders praktisch ist das Kopieren von Adressen oder ganzen Datensätzen in andere Programme, wie beispielsweise WORD.

Das «Kompendium Selbsthilfegruppen in der Onkologie» ist ein praktischer Helfer für alle, die sich mit dem Thema Krebs beschäftigen. Informationen zum Thema Krebs sind auch auf der Homepage der Deutschen Krebsgesellschaft e.V. zu finden (http://deutsche.krebsgesellschaft.de).

Das Kompendium «Selbsthilfegruppen in der Onkologie» kann gegen eine Schutzgebühr von 25,- DM bestellt werden bei der

Deutschen Krebsgesellschaft e.V.,

Paul-Ehrlich-Straße 41,

60596 Frankfurt/M.

Tel. +49 6963-00960, Fax -9130

E-mail: service@deutsche.krebsgesellschaft.de

\section{Tamoxifen schützt Frauen mit erhöhtem Brustkrebs-Risiko}

Das Medikament Tamoxifen wird seit über zwanzig Jahren zur Hormontherapie von Brustkrebs verwendet. Daß Tamoxifen auch gesunde Frauen vor Tumoren der Brust schützen kann, ist das Ergebnis einer großen, in den Vereinigten Staaten vorgenommenen Untersuchung. Mehr als 13000 Frauen haben sich daran beteiligt. Demgegenüber wurde in zwei europäischen Studien (aus Mailand und London) keine Schutzwirkung von Tamoxifen festgestellt. Die Patientenzahlen in diesen beiden Untersuchungen waren allerdings kleiner und die Risikosituation war eine andere. Daher kann nach dem derzeitigen Kenntnisstand keine generelle Empfehlung zur präventiven Einnahme von Tamoxifen ausgesprochen werden. Denn das Medikament kann selten auch schwere Nebenwirkungen haben. Sie erfordern eine strenge und individuelle Abwägung der Vor- und Nachteile einer Tamoxifen-Einnahme zum möglichen Schutz vor Brustkrebs.

\section{Brustkrebs - der häufigste Tumor der Frau}

Brustkrebs ist der häufigste Tumor der Frau. Pro Jahr erkranken in Deutschland etwa 45000 Frauen an einem Mammakarzinom. Die meisten Patientinnen sind zwischen 50 und 70 Jahre alt. Durchschnittlich erkrankt jede zehnte Frau bis zum 70. Lebensjahr an einem Tumor der Brust. Auch Männer können an Brustkrebs erkranken. Sie sind jedoch sehr viel seltener betroffen: Etwa einer unter 100 Männern erkrankt am Mammakarzinom. Trotz verbesserter therapeutischer Möglichkeiten sterben alljährlich rund 18000 Patienten an der bösartigen Erkrankung der Brust.

Etwa 5\% aller Brustkrebsfälle (familiärer Brustkrebs) beruhen auf angeborenen Veränderungen (Mutationen) in bestimmten, das Zellwachstum regulierenden Genen (BRCA1 oder BRCA2). Die Mutationen werden von Generation zu Generation weitergegeben. Personen, die diese veränderten Gene tragen, haben ein höheres Risiko, an Brustkrebs zu erkranken. Die Erkrankung tritt dann meist in jüngeren Jahren, das heißt vor dem 50. Lebensjahr, auf.

\section{Die amerikanische Brustkrebs-Präventions-Studie}

Die amerikanische Brustkrebs-Präventions-Studie (Breast Cancer Prevention Trial, BCPT) begann im April 1992. Die Untersuchung sollte prüfen, ob das Medikament Tamoxifen Frauen, die ein erhöhtes Brustkrebs-Risiko haben, vor der Erkrankung schützen kann. Erste Hinweise auf einen möglichen präventiven Effekt hatten vorangegangene kleinere Studien erbracht.

Insgesamt nahmen 13388 Frauen an der Präventionsstudie des National Surgical Adjuvant Breast and Bowel Project (NSABP) teil. Ausgewählt wurden Frauen, deren Erkrankungsrisiko für Brustkrebs erhöht ist:

- Frauen, die über 60 Jahre alt sind (ab diesem Alter tritt Brustkrebs besonders häufig auf)
KARGER

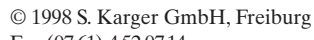


- Frauen, die über 35 Jahre alt sind und die aufgrund persönlicher Risikofaktoren (beispielsweise frühe Geschlechtsreife, keine oder späte Schwangerschaft, familiäre Belastung) ein Brustkrebs-Risiko haben, das mit dem von Frauen über 60 vergleichbar ist.

$40 \%$ der Frauen waren 35 bis 49 Jahre alt, 30\% zwischen 50 und 59 Jahren und weitere $30 \%$ über 60 Jahre.

Die Hälfte der Frauen schluckte täglich zwei Tabletten $(20 \mathrm{mg})$ Tamoxifen. Die andere Hälfte erhielt ein Placebo. Die Untersuchung war «doppelt-blind», das heißt, weder der behandelnde Arzt noch die behandelte Frau wußten, ob sie den Wirkstoff oder ein Scheinmedikament erhielt. Über die tatsächliche Verteilung war nur die Studienleitung informiert.

Ursprünglich sollte sich die Studie über 5 Jahre erstrecken. Sie wurde im Frühjahr 1998 vierzehn Monate früher als beabsichtigt abgebrochen. Eine Zwischenauswertung hatte ergeben, daß Tamoxifen das Brustkrebs-Risiko tatsächlich vermindert.

\section{Was ist Tamoxifen?}

Das Medikament Tamoxifen steht seit Mitte der 70er Jahre zur Verfügung. Es gilt heute als eine Standard-Therapie für Frauen, bei denen nach den Wechseljahren (postmenopausal) ein Brusttumor mit Hormon-Rezeptoren entdeckt wurde.

Rezeptoren sind Bindungsstellen auf der Zellmembran, der Außenhaut von Zellen. Sie können mit kleinen Antennen verglichen werden, die Botschaften von der Außenwelt der Zelle aufnehmen. An Hormon-Rezeptoren binden sind Hormone. Der Rezeptor leitet die Botschaft des Hormons in das Innere der Zelle weiter. Bei Brustkrebs übt das weibliche Östrogen über seinen Hormon-Rezeptor eine wachstumsfördernde Wirkung auf die Tumorzelle aus.

Tamoxifen ist ein «Anti-Hormon» oder «Anti-Östrogen». Es wird von den Hormon-Rezeptoren der Zelle akzeptiert und kann dort andocken, weil es dem natürlichen Östrogen ähnlich sieht. Tamoxifen verfügt aber nicht über die wachstumsfördernden Eigenschaften des Östrogens. Es blockiert die Bindungsstelle für die Östrogene und kann auf diese Weise verhindern, daß die Hormone eine Zelle zu unkontrolliertem Wachstum antreiben.

Die Behandlung mit Tamoxifen ist kein Ersatz für die chirurgische Entfernung des Tumors. Sie kann aber bei hormonempfindlichen Tumoren als zusätzliche Maßnahme nach einer Operation die Heilungschancen erhöhen und die Rückfallhäufigkeit senken.

Welche Vorteile zeigte Tamoxifen in der amerikanischen Brustkrebs-Präventions-Studie?

Bei Untersuchung der Frauen, die Tamoxifen eingenommen hatten, zeigte sich, daß das Medikament die Häufigkeit von Brustkrebs um 45\% verringern konnte: In der Gruppe, die das Scheinmedikament eingenommen hatte, waren 154 Frauen an Brustkrebs erkrankt, in der Tamoxifen-Gruppe lediglich 85 .

Tamoxifen verringert auch das Risiko, an Osteoporose zu erkranken: In der Placebo-Gruppe traten bei 71 Frauen Knochenbrüche als Folge der Osteoporose auf; in der Tamoxifen-Gruppe erlitten nur 47 eine Fraktur. Dies entspricht einer Reduktion um $40 \%$.

Reports of Oncological Societies .

Mitteilungen onkologischer Gesellschaften
Ein drittes Ziel der Studie war, festzustellen, ob Tamoxifen die Häufigkeit von Herzerkrankungen positiv beeinflussen kann. Hier zeigt das Medikament keinen Effekt.

Welche Nebenwirkungen der Tamoxifen-Behandlung wurden beobachtet?

Es zeigte sich, daß die Frauen der Tamoxifen-Gruppe ein erhöhtes Risiko für drei Krankheiten hatten, die selten auftreten, aber lebensgefährlich sind.

Das Risiko war erhöht für Gebärmutterkrebs, Venenthrombosen und Lungenembolien.

Tumoren der Gebärmutter wurden bei den Frauen, die Tamoxifen eingenommen hatten, doppelt so häufig (33 Frauen) beobachtet wie in der Gruppe, die mit dem Scheinmedikament behandelt worden war (14 Frauen). Diese Krebserkrankungen wurden jedoch sämtlich in einem Frühstadium entdeckt, wiesen prognostisch günstige Eigenschaften auf und haben bisher zu keinem Todesfall geführt.

Venenthrombosen entwickelten 30 Frauen in der TamoxifenGruppe im Vergleich zu 19 Frauen in der Placebo-Gruppe.

Lungenembolien traten in der Tamoxifen-Gruppe dreimal so häufig auf wie in der Placebo-Gruppe (17 gegenüber 6 Frauen). Weitere Nebenwirkungen, die in der Tamoxifen-Gruppe bei manchen Frauen auftraten, waren Blutungsstörungen oder Wechseljahre-Symptome.

\section{Die Europäischen Brustkrebs-Präventions-Studien}

Nach der Vorabveröffentlichung der Ergebnisse des BCPT wurden die vorläufigen Resultate zweier Europäischer Studien zur Tamoxifen-Prophylaxe von Mammakarzinomen publiziert (Tab. 1). Die Mailänder Studie umfaßte 5408 Frauen, denen die Gebärmutter entfernt worden war. Die Hälfte erhielt Tamoxifen über 5 Jahre, die andere Hälfte ein Placebo. In der Studie des Royal Marsden Hospital (London) wurden 2496 Frauen untersucht, von denen ebenfalls die Hälfte Tamoxifen, die andere Hälfte ein Placebo erhielt. Beide Studien konnten insgesamt keine Verminderung der Brustkrebshäufigkeit durch Tamoxifen feststellen, allerdings zeigte sich in der Tamoxifengruppe eine gegenüber der Plazebogruppe statistisch signifikant geringere Brustkrebsrate bei Frauen mit Hormonsubstitution. Beide Studien leiden unter einer relativ hohen Rate an Studienabbrechern (Tab. 1).

\section{Mögliche Gründe für die Diskrepanz der Ergebnisse der Studien}

Für die Diskrepanz der Ergebnisse zwischen den europäischen und der amerikanischen Studie können mehrere Faktoren verantwortlich sein: Die Teilnehmerzahlen der europäischen Studien sind deutlich geringer. Die gegenüber der BCPT in den europäischen Studien höhere Rate an Studienabbrechern hat einen größeren negativen Einfluß auf die statistische Aussagekraft. Die Royal Marsden Studie enthielt signifikant mehr junge Frauen unter 50 Jahren als die US-Studie (62\% gegenüber 40\%). Die Teilnehmerinnen der Mailänder Studie hatten ein insgesamt deutlich niedrigeres Brustkrebsrisiko, die Mammakarzinom-Inzidenz in der Placebogruppe betrug nur ein Drittel derjenigen in der BCPT. Insgesamt unterschieden sich die Studien deutlich im familiären MammakarzinomRisiko (Tab. 1), 
Tab. 1. Zusammenfassung der 3 prospektiven randomisierten Studien zur Prophylaxe von Mammakarzinomen mit Tamoxifen

\begin{tabular}{|c|c|c|c|c|c|c|c|}
\hline \multirow[t]{2}{*}{ Studie } & \multirow[t]{2}{*}{$\mathrm{N}$} & \multirow[t]{2}{*}{$\begin{array}{l}\text { Frauenjahre } \\
\text { follow-up }\end{array}$} & \multirow[t]{2}{*}{$\begin{array}{l}\text { Studien- } \\
\text { abbrecher, \% }\end{array}$} & \multicolumn{2}{|c|}{ Familiäres Risiko, \% } & \multicolumn{2}{|c|}{$\begin{array}{l}\text { Mammakarzinome/ } \\
1000 \text { Frauenjahre }\end{array}$} \\
\hline & & & & $\begin{array}{l}1 \text { MaCabei } \\
\text { Verwandter } 1^{\circ} \\
<50 \mathrm{~J}\end{array}$ & $\begin{array}{l}\geq 2 \mathrm{MaCa} \\
\text { bei Ver- } \\
\text { wandter } 1^{\circ}\end{array}$ & Placebo & Tamoxifen \\
\hline ВСРТ & 13388 & 46858 & 21,6 & 57 & 19,5 & 6,6 & 3,6 \\
\hline Mailand & 5408 & 20731 & 26,3 & 18 & 2,5 & 2,3 & 2,1 \\
\hline $\begin{array}{l}\text { Royal } \\
\text { Marsden }\end{array}$ & 2494 & 12355 & 35 & 55 & 17 & 5,0 & 4,7 \\
\hline
\end{tabular}

Welche Konsequenzen ergeben sich aus den neuen Erkenntnissen?

Noch offen ist insbesondere die Frage, ob auch Frauen, die keiner Risikogruppe angehören, von einer regelmäßigen Tamoxifen-Einnahme profitieren könnten. Ungeklärt ist auch, wie lange das Medikament eingenommen werden muß. Nicht zweifelsfrei geklärt ist bislang außerdem, ob die Gabe von Tamoxifen eine echte Prophylaxe ist. Es besteht auch die Möglichkeit, daß bei den Studienteilnehmerinnen mit erhöhtem Brustkrebs-Risiko bereits Mikrokarzinome bestanden, die durch Tamoxifen frühzeitig behandelt wurden.

Die Experten bewerten die Studienergebnisse aufgrund der bislang vorliegenden Daten wie folgt:

- Für Frauen, die ein erhöhtes Brustkrebsrisiko haben, ist der Nutzen einer Behandlung mit Tamoxifen größer zu veranschlagen als die Nachteile.

- Für Frauen mit einem nur niedrigen Risiko, an Brustkrebs zu erkranken, können die Nachteile schwerer wiegen als die Vorteile.

- Aufgrund der Nebenwirkungen, die auftreten können, kann eine generelle Empfehlung zur präventiven Einnahme von Tamoxifen derzeit nicht erfolgen.
- Frauen, die fürchten, ein erhöhtes Brustkrebs-Risiko zu haben, sollten ihren Frauenarzt aufsuchen und mit ihm diese Frage besprechen, um eine individuelle Entscheidung für oder gegen eine Tamoxifen-Einnahme in sorgfältiger Abwägung der damit verbundenen Risiken zu treffen.

Derzeit wird eine große Studie vorbereitet, die in Deutschland stattfinden soll. Die Untersuchung will Tamoxifen mit einem anderen Anti-Östrogen vergleichen. Es gehört bereits einer neuen Generation von Anti-Hormonen an, die ein anderes Wirkspektrum bei möglicherweise weniger Nebenwirkungen aufweisen. Die Hoffnung ist, mit neuen Anti-Hormonen die Häufigkeit von Brustkrebs zu verringern, ohne schwere Nebenwirkungen fürchten zu müssen.

Ansprechpartner:

Professor Dr. med. Manfred Kaufmann

Klinik für Gynäkologie und Geburtshilfe

im Klinikum der Johann-Wolfgang-Goethe-Universität

Theodor-Stern-Kai 7

60590 Frankfurt am Main

Tel. +49 6963 01-5115, Fax -6317 